\title{
Power GCD and power LCM matrices defined on GCD-closed sets over unique factorization domains
}

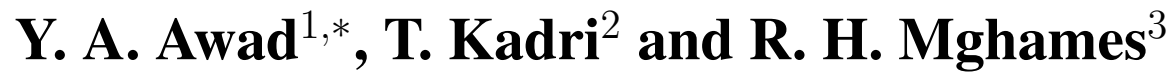 \\ ${ }^{1}$ School of Arts and Sciences, Department of Mathematics and Physics \\ Lebanese International University, Bekaa, Lebanon \\ e-mail: yehya. awadeliu.edu. lb \\ * Corresponding author. \\ ${ }^{2}$ School of Arts and Sciences, Department of Mathematics and Physics \\ Lebanese International University, Bekaa, Lebanon \\ e-mail: therrar.kadrieliu.edu.lb \\ ${ }^{3}$ School of Arts and Sciences, Department of Mathematics and Physics \\ Lebanese International University, Bekaa, Lebanon \\ e-mail: ragheb.mghames liu.edu.lb
}

Received: 10 August $2018 \quad$ Revised: 29 January $2019 \quad$ Accepted: 1 February 2019 Abstract: Let $T=\left\{t_{1}, t_{2}, \ldots, t_{m}\right\}$ be a well ordered set of $m$ distinct positive integers with $t_{1}<t_{2}<\cdots<t_{m}$. The GCD matrix on $T$ is defined as $(T)_{m \times m}=\left(t_{i}, t_{j}\right)$, where $\left(t_{i}, t_{j}\right)$ is the greatest common divisor of $t_{i}$ and $t_{j}$, and the power $G C D$ matrix on $T$ is $\left(T^{r}\right)_{m \times m}=\left(t_{i}, t_{j}\right)^{r}$, where $r$ is any real number. The $L C M$ matrix on $T$ is defined as $[T]_{m \times m}=\left[t_{i}, t_{j}\right]$, where $\left[t_{i}, t_{j}\right]$ is the least common multiple of $t_{i}$ and $t_{j}$, and the power $L C M$ matrix on $T$ is $\left[T^{r}\right]_{m \times m}=\left[t_{i}, t_{j}\right]^{r}$. Set $T=\left\{t_{1}, t_{2}, \ldots, t_{m}\right\}$ is said to be gcd-closed if $\left(t_{i}, t_{j}\right) \in T$ for every $t_{i}$ and $t_{j}$ in $T$. In this paper, we give a generalization for the power GCD and $L C M$ matrices defined on gcdclosed sets over unique factorization domains (UFDs). Moreover, we present a speculation for a generalization of Bourque-Ligh conjecture to UFDs which states that the least common multiple matrix defined on a gcd-closed $P$-ordered set in any UFD is nonsingular. Some examples that show what is done are additionally given in $Z[i]$ and $Z_{p}[x]$.

Keywords: Power GCD P-matrix, Power $L C M P$-Matrix, $P$-ordering, gcd-closed sets, Prime residue system, Unique factorization domains.

2010 Mathematics Subject Classification: Primary 11C20, 11A25; Secondary 13F15, 15A36, $16 \mathrm{U} 30$. 


\section{Introduction}

Let $T=\left\{t_{1}, t_{2}, \ldots, t_{m}\right\}$ be a well-ordered set of $m$ distinct positive integers such that $t_{1}<t_{2}<\cdots<t_{m}$. The GCD matrix on $T$ is defined as $(T)_{m \times m}=\left(t_{i}, t_{j}\right)$, where $\left(t_{i}, t_{j}\right)$ is the greatest common divisor of $t_{i}$ and $t_{j}$, and the $L C M$ matrix on $T$ is defined as $[T]_{m \times m}=\left[t_{i}, t_{j}\right]$, where $\left[t_{i}, t_{j}\right]$ is the least common multiple of $t_{i}$ and $t_{j}$. Moreover, set $T$ is said to be:

- factor-closed if for every $t_{i} \in T$, all the divisors of $t_{i}$ are also in $T$.

- gcd-closed if the greatest common divisor $\left(t_{i}, t_{j}\right) \in T$ for all elements $t_{i}$ and $t_{j}$ in $T$.

- lcm-closed if the least common multiple $\left[t_{i}, t_{j}\right] \in T$ for all elements $t_{i}$ and $t_{j}$ in $T$.

In $1875 / 76$, Smith [18] showed that if $T=\{1,2, \ldots, m\}$, then the determinant of the $G C D$ matrix $(T)$ defined on $T$ is equal to the product $\phi(1) \phi(2) \cdots \phi(m)$ and the determinant of the $L C M$ matrix $[T]$ defined on $T$ is also equal to $\phi(1) \pi(1) \phi(2) \pi(2) \cdots \phi(m) \pi(m)$, where $\phi$ is the Euler's totient function and $\pi$ is a multiplicative function for prime powers. Also, he showed that if $T=\left\{t_{1}, t_{2}, \ldots, t_{m}\right\}$ is a factor-closed set of distinct positive integers, then $\operatorname{det}(T)=$ $\phi\left(t_{1}\right) \phi\left(t_{2}\right) \cdots \phi\left(t_{m}\right)$ and $\operatorname{det}[T]=\phi\left(t_{1}\right) \pi\left(t_{1}\right) \phi\left(t_{2}\right) \pi\left(t_{2}\right) \cdots \phi\left(t_{m}\right) \pi\left(t_{m}\right)$. Moreover, Smith showed that if $f$ is an arithmetical multiplicative function, then $\operatorname{det}[f(i, j)]=(f * \mu)(1)(f * \mu)(2) \cdots$ $(f * \mu)(m)$, where $\mu$ is the Möbius function and $(f * \mu)$ is the Dirichlet convolution of $f$ and $\mu$. In 1988/89, S. Beslin and S. Ligh [2, 4, 17], showed that if $(T)$ is defined on a factor-closed set $T$, then it could be written as a product of a matrix $B$ and its corresponding transpose $B^{T}$. Moreover, they conjectured that if $T=\left\{t_{1}, t_{2}, \ldots, t_{m}\right\}$ is a set of distinct positive integers, then $\operatorname{det}(T)=\phi\left(t_{1}\right) \phi\left(t_{2}\right) \cdots \phi\left(t_{m}\right)$ if and only if $T$ is factor-closed. In 1989, Beslin and El-Kassar [1] exposed $G C D$ matrices over unique factorization domains (UFDs). In 1990, Z. Li [16] gave a generalization for Smith's determinant by obtaining the value of $(T)$ defined on an arbitrary ordered set of distinct positive integers, and he showed that the conjecture of Beslin-Ligh is true. In 1991/92, Beslin and Ligh [3,5] proved that if $T=\left\{t_{1}, t_{2}, \ldots, t_{m}\right\}$ is a gcd-closed set, then $(T)$ could be factored as a product of two triangular matrices and they calculated its determinant. In addition, they gave a structure theorem for the $L C M$ matrix $[T]$ and showed that it is non-singular. In the same year, K. Bourque and S. Ligh [6] provided a formula for the inverse of $(T)$ and $[T]$ on factor-closed sets, and showed that $\operatorname{det}(T)$ divides $\operatorname{det}[T]$. In addition, they also conjectured that if $[T]$ is defined on a gcd-closed set, then it is non-singular (Bourque-Ligh Conjecture). In 1996/97, P. Haukkanen et al. [10, 11] presented a brief review of papers relating to Smith's determinant and pointed out a common structure that could be found in many extensions and analogues of Smith's determinant. Further, they gave a counterexample for the conjecture of Bourque and Ligh that the least common multiple matrix on any gcd-closed set is invertible. Moreover, they calculated the $G C D$ and $L C M$ matrices for lcm-closed and gcd-closed sets. In 1998, Hong [12, 13, 14] showed that the Bourque-Ligh conjecture is true for a certain class of gcd-closed sets. In addition, he showed new bounds for $\operatorname{det}\left[f\left(x_{i}, x_{j}\right)\right]$ for a certain class of arithmetical functions and semi-multiplicative functions, which improve the results obtained by Bourque and Ligh [6]. Later in 2009/10, El-Kassar et al. [8, 9] presented generalizations for the 
$G C D$ and $L C M$ matrices defined on both factor-closed and gcd-closed sets in principal ideal domains (PIDs).

Now, let $r$ be a real number and let $T=\left\{t_{1}, t_{2}, \ldots, t_{m}\right\}$ be a well ordered set of $m$ distinct positive integers such that $t_{1}<t_{2}<\cdots<t_{m}$. The power GCD matrix defined on $T$ is the $m \times m$ matrix $\left(T^{r}\right)=\left(t_{i j}\right)_{m \times m}$ such that $t_{i j}=\left(t_{i}, t_{j}\right)^{r}$, where $\left(t_{i}, t_{j}\right)$ is the greatest common divisor of $t_{i}$ and $t_{j}$. The power $L C M$ defined on $T$ is the $m \times m$ matrix $\left[T^{r}\right]=\left(t_{i j}\right)_{m \times m}$ such that $t_{i j}=\left[t_{i}, t_{j}\right]^{r}$, where $\left[t_{i}, t_{j}\right]$ is the least common multiple of $t_{i}$ and $t_{j}$. In 1996, S. Z. Chun [7] introduced the concept of $r$-th power of the GCD and $L C M$ matrices $\left(T^{r}\right)$ and $\left[T^{r}\right]$ respectively, where $r$ is a real number. Moreover, he calculated $\operatorname{det}\left(T^{r}\right)$ and $\operatorname{det}\left[T^{r}\right]$ over the factor-closed set $T$. In addition, he discussed $\left(T^{r}\right)$ and $\left[T^{r}\right]$ over gcd-closed sets and obtained the structures for their inverses and reciprocals over the domain of natural numbers. In 2009, S. Hong et al. [15] presented the structures, determinants, and the non-singularity of the power $G C D$ matrices for a UFD by considering the results of Beslin and El-Kassar [1].

In this paper, we give a generalization for the power $G C D$ and $L C M P$-matrices characterized on gcd-closed sets over unique factorization domains (UFDs). Additionally, we make a speculation for Bourque-Ligh conjecture to UFDs. A few precedents on $Z[i]$ and $Z_{p}[x]$ that show what as been done are additionally given. The article comprises of six particular sections. In the first section, we present the essentials and the definitions required all through our work. In the second and the third sections, we present structure hypotheses and the determinants of the power $G C D P$-matrices and their reciprocals characterized on gcd-closed $P$-ordered sets in unique factorization domains. In addition, a few precedents were given in $Z_{2}[x]$. In the fourth section, we study the inverses of the power GCD P-matrices on gcd-closed sets over UFDs. Finally, in the fifth and the sixth sections, the structure hypotheses and the determinants of the power $L C M$ $P$-matrices characterized on gcd-closed $P$-ordered sets in UFDs are presented. Additionally, a few precedents were given in $Z_{2}[x]$ and $Z[i]$.

\section{Preliminaries and definitions}

It is well-known from literature that a zero-divisor is a non-zero element $s$ of a ring $R$ such that there exists another non-zero element $t$ in $R$ with $s t=0$. An integral domain $D$ is a commutative ring with unity and no zero-divisors. A subring $A$ of a ring $R$ with unity is called two-sided ideal if for every $s \in R$ and $a \in A$ both $s a$ and $a s$ are also in $A$. Moreover, two non-zero elements $s$ and $t$ of an integral domain $D$ are said to be associates if $s \approx u t$ where $u$ is a unit in $D$.

Definition 1. A well-ordered set $P \approx\left\{p_{1}, p_{2}, \ldots, p_{n}\right\}$ of non-zero and non-associate prime elements of an integral domain $R$ is called a prime residue system of $R$ if $P$ is complete and every prime element of $R$ either belongs to $P$, or is an associate to an element of $P$.

Definition 2. A unique factorization domain (UFD) is defined to be an integral domain $R$ in which every non-zero element $t$ of $R$ can be written uniquely up to order and associates as a product (an empty product if $t$ is a unit) of prime elements $p_{i}$ of $R$ and $a$ unit $u$. 
That is, $R$ is said to be a unique factorization domain of prime residue system $P \approx\left\{p_{1}, p_{2}, \ldots, p_{n}\right\}$ if any non-zero element $t \in R$ could be written as $t \approx u p_{1}^{\alpha_{1}} p_{2}^{\alpha_{2}} \cdots p_{m}^{\alpha_{m}}$, where $p_{j}$ are distinct elements of $P$, the $\alpha_{j}$ are positive integers and $u$ is a unit in $R$.

Definition 3. An element $t \in R$ is called a P-number if $t$ is a product of elements from the prime residue system $P$.

In our work, we denote by $\bar{P}$ to be the set of 1 and all $P$-numbers. Moreover, we call $d$ a $P$-divisor of $t$ in $R$ if $d$ divides $t$ and $d \in \bar{P}$. If $t$ and $s$ are both non-zero elements in $R$, then we use $(t, s)_{p}$ to denote the greatest common $P$-divisor of $t$ and $s$ in $R$.

Definition 4. Let $R$ be a UFD and $P \approx\left\{p_{1}, p_{2}, \ldots, p_{i}, \ldots\right\}$ be a complete prime residue system of $R$. The generalized Jordan totient function $\tilde{s}_{k, R}$ over a unique factorization domain $R$ is defined to be the multiplicative function defined on $R-\{0\}$ such that

$$
\tilde{s}_{k, R}(t) \approx \prod_{i \approx 1}^{m} p_{i}^{k\left(\alpha_{i}-1\right)}\left(p_{i}^{k}-1\right) .
$$

Note that if $t$ is a unit then $\tilde{s}_{k, R}(t) \approx 1$. Moreover, $\tilde{s}_{k, R}\left(t_{i} t_{j}\right) \approx \tilde{s}_{k, R}\left(t_{i}\right) \tilde{s}_{k, R}\left(t_{j}\right)$ for any two relatively prime elements $t_{i}$ and $t_{j}$ in $T$.

Theorem 1. Let $P$ be a prime residue system of a UFD $R$, and let $t$ be an element of $T$, and let $k$ be a positive integer. If $E(t)$ is a complete set of distinct non-associate divisors $d$ of $t$ in $T$, then

$$
t^{k} \approx \sum_{d \in E(t)} \tilde{s}_{k, R}(d)
$$

Proof. Since $\tilde{s}_{k, s}$ is multiplicative, then the function $f(t) \approx \sum_{d \in E(t)} \tilde{s}_{k, R}(d)$ is also multiplicative. Hence, for any $p_{j} \in P$,

$$
\begin{aligned}
f\left(p_{i}^{n_{i}}\right) & \approx \sum_{d \in E\left(p_{i}^{n_{i}}\right)} \tilde{s}_{k, R}(d) \\
& \approx 1+\left(p_{i}\right)^{k(1-1)}\left(p_{i}{ }^{k}-1\right)+\left(p_{i}\right)^{k(2-1)}\left(p_{i}{ }^{k}-1\right)+\cdots+\left(p_{i}\right)^{k\left(n_{i}-1\right)}\left(p_{i}{ }^{k}-1\right) \\
& \approx 1+\left(p_{i}\right)^{k}-1+\left(p_{i}\right)^{k(2-1)}-\left(p_{i}\right)^{k}+\cdots+\left(p_{i}\right)^{k n_{i}}-\left(p_{i}\right)^{k\left(n_{i}-1\right)} \\
& \approx\left(p_{i}\right)^{k n_{i}} \\
& \approx\left(p_{i}^{n_{i}}\right)^{k} .
\end{aligned}
$$

Now, if $t$ is a product of non-zero non-associate elements from $P$, then $f(t) \approx \sum_{d \in E(t)} \widetilde{s}_{k, R}(d)$. The proof is complete.

Definition 5. ( $P$-Ordering in UFDs) The P-ordering in a UFD $R$ is defined via the following scheme: $t_{i}<_{p} t_{j}$ iffor some $m, k_{i_{m}}<k_{j_{m}}$ and $k_{i_{c}}=k_{j_{c}}$ for each $c<m$ and this relation $<_{p}$ is considered as a well-defined linear ordering on $T$.

Hence, if the ordered set $T$ is such that $t_{1}<_{p} t_{2}<_{p}<\cdots<_{p} t_{m}$, we say that $T$ is $P$-ordered and the matrix defined on $T$ is a $P$-matrix.

1992, K. Bourque and S. Ligh [6] calculated the determinant of $[T]$ when $T$ is gcd-closed, and they made the following conjecture.

Bourque-Ligh Conjecture. The LCM matrix on a gcd-closed set is invertible. 


\section{Power $G C D$ matrices on $G C D$-closed sets over a UFD}

Let $R$ be a UFD and $P$ be a complete prime residue system of $R$. If $T \approx\left\{t_{1}, t_{2}, \ldots, t_{n}\right\}$ is a $P$-ordered set of non-zero non-associate elements in $R$. In the following, we present structure theorems of the power $G C D P$-matrices, and their determinants on gcd-closed $P$-ordered sets over a UFD. Moreover, some examples are given in $Z_{2}[x]$.

Definition 6. Let $R$ be a UFD and $P \approx\left\{p_{1}, p_{2}, \ldots, p_{i}, \ldots\right\}$ be a complete prime residue system of $R$. Let $T \approx\left\{t_{1}, t_{2}, \ldots, t_{m}\right\}$ be a P-ordered set of non-zero non-associate elements in $R$. Then, $T$ is said to be gcd-closed iffor every $t_{i}$ and $t_{j}$ in $T$ their greatest common divisor $\left(t_{i}, t_{j}\right)_{p}$ is an associate to some element $t_{k} \in T$.

Definition 7. Let $R$ be a UFD and $P \approx\left\{p_{1}, p_{2}, \ldots, p_{i}, \ldots\right\}$ be a complete prime residue system of $R$. If $T \approx\left\{t_{1}, t_{2}, \ldots, t_{m}\right\}$ is a P-ordered set of non-zero non-associate elements in $R$ and $r \geq 1$ is an integer, then the powers GCD P-matrix defined on $T$ is the $m \times m$ matrix $\left[T^{r}\right]=$ $\left(t_{i j}\right)_{m \times m}$ such that $t_{i j} \approx\left(t_{i}, t_{j}\right)_{p}{ }^{r}$.

\subsection{Factorizations of power $G C D P$-matrices on $G C D$-closed sets over a UFD}

Theorem 2. Let $R$ be a UFD and $P \approx\left\{p_{1}, p_{2}, \ldots, p_{i}, \ldots\right\}$ be a complete prime residue system of $R$. If $T \approx\left\{t_{1}, t_{2}, \ldots, t_{m}\right\}$ is a P-ordered set of non-zero non-associate elements in $R$, then

$$
\left(T^{r}\right)_{p} \approx E A_{r} E^{T}
$$

Proof. Let $D=\left\{y_{1}, y_{2}, \ldots, y_{n}\right\}$ be the smallest gcd-closed set containing $T$ in $R$, and let $E(x)$ be a complete set of distinct non-associate divisors $d$ of $t$ in $R$. Define the $n \times n$ diagonal matrix $A_{r}$ as:

$$
A_{r}=\operatorname{diag}\left(\sum_{\substack{d \in E\left(y_{1}\right), d \notin E\left(y_{u}\right) \\ y_{u}<p}} \tilde{s}_{r, s}(d), \sum_{\substack{d \in E\left(y_{2}\right), d \notin E\left(y_{u}\right) \\ y_{u}<p}} \tilde{s}_{r, s}(d), \ldots, \sum_{\substack{d \in E\left(y_{n}\right), d \notin E\left(y_{u}\right) \\ y_{u}<p}} \tilde{s}_{r, s}(d)\right)
$$

and $E=\left(e_{i j}\right)_{m \times n}$ such that

$$
e_{i j}= \begin{cases}1, & \text { if } y_{j} \in E\left(t_{i}\right) \\ 0, & \text { otherwise }\end{cases}
$$

Then,

$$
\left(E A_{r} E^{T}\right)_{i j} \approx \sum_{k=1}^{n}\left(e_{i k}\left(\sum_{\begin{array}{l}
d \in E\left(y_{k}\right) \\
d \notin E\left(y_{u}\right) \\
y_{u}<p
\end{array} y_{k}} \tilde{s}_{r, s}(d)\right) e_{j k}\right) \approx \sum_{y_{k} \in E\left(t_{i}\right)}\left(\sum_{\substack{d \in E\left(y_{k}\right) \\
d \notin E\left(y_{u}\right) \\
y_{k} \in E\left(t_{j}\right)}} \tilde{s}_{r, s}(d)\right)
$$




$$
\approx \sum_{y_{k} \in E\left(\left(t_{i}, t_{j}\right)_{p}\right)}\left(\sum_{\substack{d \in E\left(y_{k}\right), d \notin E\left(y_{u}\right), y_{u}<p}} \tilde{s}_{r, s}(d)\right) \approx\left(t_{i}, t_{j}\right)_{p}{ }^{r} .
$$

Example 1. $T=\left\{1,1+x, 1+x^{3}\right\}$ is an ordered set in $\mathbb{Z}_{2}[x]$. Then,

$$
\begin{aligned}
E A_{2} E^{T} & \approx\left[\begin{array}{lll}
1 & 0 & 0 \\
1 & 1 & 0 \\
1 & 1 & 1
\end{array}\right]\left[\begin{array}{ccc}
1 & 0 & 0 \\
0 & x^{2} & 0 \\
0 & 0 & x^{2}+x^{6}
\end{array}\right]\left[\begin{array}{lll}
1 & 1 & 1 \\
0 & 1 & 1 \\
0 & 0 & 1
\end{array}\right] \\
& \approx\left[\begin{array}{ccc}
1 & 1 & 1 \\
1 & 1+x^{2} & 1+x^{2} \\
1 & 1+x^{2} & 1+x^{6}
\end{array}\right] \approx\left(T^{2}\right)_{p} .
\end{aligned}
$$

Theorem 3. Let $R$ be a UFD and $P \approx\left\{p_{1}, p_{2}, \ldots, p_{i}, \ldots\right\}$ be a complete prime residue system of $R$. If $T \approx\left\{t_{1}, t_{2}, \ldots, t_{m}\right\}$ is a P-ordered set of non-zero non-associate elements in $R$, then

$$
\left(T^{r}\right)_{p} \approx A_{r} B_{r}
$$

where $\left[A_{r}\right]_{n \times m}$ and $\left[B_{r}\right]_{m \times n}$ for some positive integer $n \geq m$.

Proof. Let $D=\left\{y_{1}, y_{2}, \ldots, y_{n}\right\}$ be the smallest gcd-closed set containing $T$ in $R$, and $E(x)$ be a complete set of distinct non-associate divisors $d$ of $t$ in $R$. Define the matrix $A_{r}=\left(a_{i j}\right)_{m \times n}$ as:

$$
a_{i j}=\left\{\begin{array}{cl}
\sum_{\substack{d \in E\left(y_{j}\right), d \notin E\left(y_{u}\right) \\
y_{u}<<_{p} y_{j}}} \tilde{s}_{r, s}(d), & \text { if } y_{j} \in E\left(t_{i}\right) \\
0, & \text { otherwise }
\end{array}\right.
$$

and $B_{r}=\left(b_{i j}\right)$ as:

$$
b_{i j}=\left\{\begin{array}{ll}
1, & \text { if } a_{j i} \neq 0 \\
0, & \text { if } a_{j i}=0
\end{array} .\right.
$$

Therefore,

$$
\begin{aligned}
\left(A_{r} B_{r}\right)_{i j} & \approx \sum_{k=1}^{n}\left(a_{i k} b_{k j}\right) \approx \sum_{\substack{y_{k} \in E\left(t_{i}\right) \\
y_{k} \in E\left(t_{j}\right)}}\left(\sum_{\substack{d \in E\left(y_{k}\right), d \notin E\left(y_{u}\right) \\
y_{u}<_{p} y_{k}}} \tilde{s}_{r, s}(d)\right) \\
& \approx \sum_{y_{k} \in E\left(\left(t_{i}, t_{j}\right)_{p}\right)}\left(\sum_{\substack{d \in E\left(y_{k}\right), d \notin E\left(y_{u}\right) \\
y_{u}<p}} \tilde{s}_{r, s}(d)\right) \approx\left(t_{i}, t_{j}\right)_{p}{ }^{r} .
\end{aligned}
$$


Example 2. Let $T \approx\left\{1,1+x, 1+x^{3}\right\}$ in $\mathbb{Z}_{2}[x]$, then

$$
\begin{aligned}
A_{2} B_{2} & \approx\left[\begin{array}{ccc}
1 & 0 & 0 \\
1 & x^{2} & 0 \\
1 & x^{2} & x^{2}+x^{6}
\end{array}\right]\left[\begin{array}{lll}
1 & 1 & 1 \\
0 & 1 & 1 \\
0 & 0 & 1
\end{array}\right] \\
& \approx\left[\begin{array}{ccc}
1 & 1 & 1 \\
1 & 1+x^{2} & 1+x^{2} \\
1 & 1+x^{2} & 1+x^{6}
\end{array}\right] \approx\left(T^{2}\right)_{p} .
\end{aligned}
$$

Theorem 4. Let $R$ be a UFD and $P \approx\left\{p_{1}, p_{2}, \ldots, p_{i}, \ldots\right\}$ be a complete prime residue system of $R$. If $T \approx\left\{t_{1}, t_{2}, \ldots, t_{m}\right\}$ is a P-ordered set of non-zero non-associate elements in $R$, then

$$
\left(T^{r}\right)_{p} \approx A_{r} A_{r}^{T}
$$

Proof. Let $\bar{F}$ be an extension of the field of fractions $F$ of $R$ in which $\widetilde{s}_{r, s}(t)$ has square roots for every $t_{i} \in T, D=\left\{y_{1}, y_{2}, \ldots, y_{n}\right\}$ be the smallest gcd-closed set containing $T$ in $R$, and $E(x)$ be a complete set of distinct non-associate divisors $d$ of $t$ in $R$. Define the matrix $A_{r}$ as:

$$
a_{i j}=\left\{\begin{array}{cl}
\sqrt{\sum_{d \in E\left(y_{j}\right)} \tilde{s}_{r, s}(d)}, & \text { if } y_{j} \in E\left(t_{i}\right) \\
d \notin E\left(y_{u}\right) \\
y_{u}<p y_{j} & \text { otherwise } \\
0, & .
\end{array} .\right.
$$

Then,

$$
\begin{aligned}
& \left(A_{r} A_{r}^{T}\right)_{i j} \approx \sum_{k=1}^{n}\left(a_{i k} a_{j k}\right) \approx \sum_{\substack{y_{k} \in E\left(t_{i}\right) \\
y_{k} \in E\left(t_{j}\right)}}\left(\sqrt{\sum_{\substack{d \in E\left(y_{k}\right) \\
d \notin E\left(y_{u}\right) \\
y_{u}<p y_{k}}} \tilde{s}_{r, s}(d)} \sqrt{\sum_{\substack{d \in E\left(y_{k}\right) \\
d \notin E\left(y_{u}\right) \\
y_{u}<p}} \tilde{s}_{r, s}(d)}\right) \\
& \approx \sum_{y_{k} \in E\left(\left(t_{i}, t_{j}\right)_{p}\right)}\left(\sum_{\substack{d \in E\left(y_{k}\right) \\
d \notin E\left(y_{u}\right) \\
y_{u}<p}} \widetilde{s}_{r, s}(d)\right) \approx\left(t_{i}, t_{j}\right)_{p}{ }^{r} .
\end{aligned}
$$

Example 3. If $T \approx\left\{1,1+x, 1+x^{3}\right\}$ in $\mathbb{Z}_{2}[x]$, then

$$
\begin{aligned}
A_{2} A_{2}^{T} & \approx\left[\begin{array}{ccc}
1 & 0 & 0 \\
1 & \sqrt{x^{2}} & 0 \\
1 & \sqrt{x^{2}} & \sqrt{x^{2}+x^{6}}
\end{array}\right]\left[\begin{array}{ccc}
1 & 1 & 1 \\
0 & \sqrt{x^{2}} & \sqrt{x^{2}} \\
0 & \sqrt{x^{2}} & \sqrt{x^{2}+x^{6}}
\end{array}\right] \\
& \approx\left[\begin{array}{ccc}
1 & 1 & 1 \\
1 & 1+x^{2} & 1+x^{2} \\
1 & 1+x^{2} & 1+x^{6}
\end{array}\right] \approx\left(T^{2}\right)_{p} .
\end{aligned}
$$




\subsection{Determinants of power $G C D P$-matrices}

\section{on $G C D$-closed sets over a UFD}

Theorem 5. Let $R$ be a UFD and $P \approx\left\{p_{1}, p_{2}, \ldots, p_{i}, \ldots\right\}$ be a complete prime residue system of $R$. If $T \approx\left\{t_{1}, t_{2}, \ldots, t_{m}\right\}$ is a P-ordered gcd-closed set of non-zero non-associate elements in $R$, then

$$
\operatorname{det}\left(T^{r}\right)_{p} \approx \prod_{i=1}^{m}\left(\sum_{\substack{d \in E\left(t_{i}\right), d \notin E\left(t_{u}\right) \\ t_{u}<_{p} t_{i}}} \widetilde{s}_{r, s}(d)\right)
$$

Proof. First Proof: By Theorem 1, we have $\left(T^{r}\right)_{p} \approx E A_{r} E^{T}$ and since $T$ is a gcd-closed set, then $T \approx D \approx\left\{y_{1}, y_{2}, \ldots, y_{m}\right\}$ and $E$ is a lower triangular matrix with diagonal entries $e_{i j} \approx 1$ so that $\operatorname{det}[E]=1$. Thus,

$$
\operatorname{det}\left(T^{r}\right)_{p} \approx \operatorname{det}\left(E A_{r} E^{T}\right) \approx \prod_{i=1}^{m}\left(\sum_{\substack{d \in E\left(t_{i}\right), d \notin E\left(t_{u}\right) \\ t_{u}<_{p} t_{i}}} \widetilde{s}_{r, s}(d)\right)
$$

Second Proof: By Theorem 2, we have $\left(T^{r}\right)_{p} \approx A_{r} B_{r}$ and since $T$ is gcd-closed in $R$, then $A_{r}$ and $B_{r}$ are lower and upper triangular matrices, respectively, with respective diagonal entries $a_{i i}=\sum_{\substack{d \in E\left(t_{i}\right), d \notin E\left(t_{u}\right) \\ t_{u}<t_{p}}} \tilde{s}_{r, s}(d)$ and $b_{i i}=1$. Therefore,

$$
t_{u}<{ }_{p} t_{i}
$$

$$
\operatorname{det}\left(T^{r}\right)_{p} \approx \operatorname{det}\left(A_{r}\right) \operatorname{det}\left(B_{r}\right) \approx \operatorname{det}\left(A_{r}\right) \approx \prod_{i=1}^{m}\left(\sum_{\substack{d \in E\left(t_{i}\right), d \notin E\left(t_{u}\right) \\ t_{u}<_{p} t_{i}}} \tilde{s}_{r, s}(d)\right) .
$$

Third Proof: By Theorem 3, we have $\left(T^{r}\right)_{p} \approx A_{r} A_{r}^{T}$ and since $T$ is a gcd-closed set in $R$, then $A_{r}$ is a lower matrix with diagonal entries $a_{i i}=\sqrt{\sum_{\substack{d \in E\left(t_{i}\right), d \notin E\left(t_{u}\right) \\ t_{u}<p} t_{i}} \tilde{s}_{r, s}(d)}$ for all $1 \leq i \leq m$. Then,

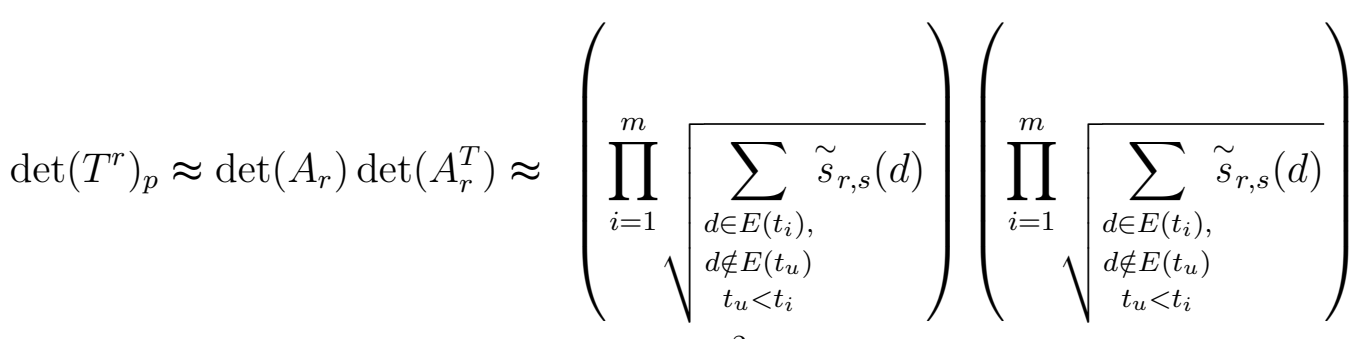

$$
\begin{aligned}
& \approx \prod_{i=1}^{m}\left(\sqrt{\sum_{\substack{d \in E\left(t_{i}\right), d \notin E\left(t_{u}\right) \\
t_{u}<t_{i}}} \tilde{s}_{r, s}(d)}\right)^{2} \approx \prod_{i=1}^{m}\left(\sum_{\begin{array}{c}
d \in E\left(t_{i}\right), d \notin E\left(t_{u}\right) \\
t_{u}<t_{i}
\end{array}} \tilde{s}_{r, s}(d)\right) .
\end{aligned}
$$


Corollary 1. (Beslin-Ligh Result) If $T=\left\{t_{1}, t_{2}, \ldots, t_{m}\right\}$ is a gcd-closed set of positive integers, then

$$
\operatorname{det}\left(T^{r}\right)_{p}=\prod_{i=1}^{m}\left(\sum_{d / t_{i}, d \nmid t_{t}, t_{t}<t_{i}}(\phi(d))\right)
$$

Proof. Using Theorem 4 by taking $r=1, R=\mathbb{Z}$, then $<_{p}$ is the usual $<$ in $\mathbb{Z}$ and $\tilde{s}_{1, \mathbb{Z}}=\phi$. Therefore,

$$
\begin{aligned}
\operatorname{det}\left(T^{r}\right)_{p} & =\prod_{i=1}^{m}\left(\sum_{\left.\begin{array}{l}
d \in E\left(t_{i}\right) \\
d \notin E\left(t_{u}\right) \\
t_{u}<p t_{i}
\end{array}\right)} \tilde{s}_{r, s}(d)=\prod_{i=1}^{m}\left(\sum_{\left.\begin{array}{l}
d \in E\left(t_{i}\right) \\
d \notin E\left(t_{u}\right) \\
t_{u}<p t_{i}
\end{array}\right)} \tilde{s}_{1, \mathbb{Z}}(d)\right)\right. \\
& =\prod_{i=1}^{m}\left(\sum_{d / t_{i}, d t_{t}, t_{t}<t_{i}}(\phi(d))\right) .
\end{aligned}
$$

Definition 8. Let $R$ be a UFD and $P \approx\left\{p_{1}, p_{2}, \ldots, p_{m}\right\}$ be a complete prime residue system of $R$. If $T \approx\left\{t_{1}, t_{2}, \ldots, t_{m}\right\}$ is a P-ordered set of non-zero non-associate elements in $R$ and $t \in S$ such that $t \approx u p_{1}^{\alpha_{1}} p_{2}^{\alpha_{2}} \cdots p_{m}^{\alpha_{m}}$, where $u$ is a unit in $S, p_{i}$ are distinct elements of $P$, and $\alpha_{i}$ are positive integers for all $1 \leq i \leq m$, then we define the multiplicative function $\Phi_{s}$ on $R /\{0\}$ as:

$$
\Phi_{s}(t) \approx \prod_{i=1}^{m} p_{i}^{\alpha_{i}-1}\left(p_{i}-1\right)
$$

We note that $\Phi_{s}(t) \approx 1$ if and only if $t$ is a unit in $R$. Moreover, $\Phi_{s}(x y) \approx \Phi_{s}(x) \Phi_{s}(y)$ for any two relatively prime elements $x$ and $y$ in $R$.

Theorem 6. Let $R$ be a UFD and $P \approx\left\{p_{1}, p_{2}, \ldots, p_{m}\right\}$ be a complete prime residue system of $R$. If $T \approx\left\{t_{1}, t_{2}, \ldots, t_{m}\right\}$ is a P-ordered set of non-zero non-associate elements in $R$, and $E(t)$ is a complete set of distinct non-associate divisors $d$ of $t$ in $R$, then

$$
t \approx \sum_{d \in E(t)} \Phi_{s}(d)
$$

Proof. Since $\Phi_{s}$ is multiplicative, then

$$
f(t) \approx \sum_{d \in E(t)} \Phi_{s}(d)
$$

is also multiplicative. So, for any $p_{j}$ in the prime residue system $P$ in $R$ we have

$$
f\left(p_{i}^{n_{i}}\right) \approx \sum_{d \in E\left(p_{i}^{n_{i}}\right)} \Phi_{s}(d) \approx 1+\left(p_{i}-1\right)+\left(p_{i}^{2}-p_{i}\right)+\cdots+\left(p_{i}^{n_{i}}-p_{i}^{n_{i}-1}\right) \approx p_{i}^{n_{i}} .
$$


Now, if $t \approx u p_{1}^{\alpha_{1}} p_{2}^{\alpha_{2}} \cdots p_{m}^{\alpha_{m}}$, then

$$
\sum_{d \in E(t)} \Phi_{s}(d) \approx f(t) \approx \prod_{i=1}^{m}\left(\sum_{d \in E\left(p_{i}^{n_{i}}\right)} \Phi_{s}(d)\right) \approx u \prod_{i=1}^{m} p_{i}^{n_{i}} \approx t .
$$

Remark 1. Let $R$ be a UFD and $P \approx\left\{p_{1}, p_{2}, \ldots, p_{m}\right\}$ be a complete prime residue system of $R$. If $T \approx\left\{t_{1}, t_{2}, \ldots, t_{m}\right\}$ is a P-ordered set of non-zero non-associate elements in $R$, then it is clear that if we take $r=1$ and $\tilde{s}_{1, R} \approx \Phi_{R}$ we have a generalization for Beslin-Ligh Result [5] over a UFD. That is;

$$
\operatorname{det}(T)_{p} \approx \prod_{i=1}^{m}\left(\sum_{d \in E\left(t_{i}\right), d \notin E\left(t_{u}\right), t_{u}<t_{i}} \Phi_{s}(d)\right)
$$

\section{Reciprocal power $G C D P$-matrices on $G C D$-closed sets over a UFD}

Definition 9. Let $R$ be a UFD and $P \approx\left\{p_{1}, p_{2}, \ldots, p_{i}, \ldots\right\}$ be a complete prime residue system of $R$. If $T \approx\left\{t_{1}, t_{2}, \ldots, t_{m}\right\}$ is a P-ordered set of non-zero non-associate elements in $R$, the $m \times m$ matrix $1 /\left(T^{r}\right)_{p}=\left(t_{i j}\right)_{m \times m}$ such that $\left(t_{i j}\right) \approx\left(t_{i}, t_{j}\right)_{p}{ }^{-r} \approx \frac{1}{\left[\left(t_{i}, t_{j}\right)_{p}\right]^{r}}$ is called the reciprocal power GCD P-matrix defined on T over a UFD $R$.

\subsection{Factorizations of reciprocal power $G C D P$-matrices on $G C D$-closed sets over a UFD}

Theorem 7. Let $R$ be a UFD and $P \approx\left\{p_{1}, p_{2}, \ldots, p_{i}, \ldots\right\}$ be a complete prime residue system of $R$. If $T \approx\left\{t_{1}, t_{2}, \ldots, t_{m}\right\}$ is a P-ordered set of non-zero non-associate elements in $R$, then

$$
1 /\left(T^{r}\right)_{p} \approx E A_{-r} E^{T}
$$

Proof. Let $D=\left\{y_{1}, y_{2}, \ldots, y_{n}\right\}$ be the smallest gcd-closed set containing $T$ in the principle ideal domain $S$ and $E(t)$ be a complete set of distinct non-associate divisors $d$ of $t$ in $R$. Define the $n \times n$ diagonal matrix $A_{-r}$ as:

$$
A_{-r}=\operatorname{diag}\left(\sum_{\substack{d \in E\left(y_{1}\right) \\ d \notin E\left(y_{u}\right) \\ y_{u}<y_{1}}} \tilde{s}_{-r, s}(d), \sum_{\substack{d \in E\left(y_{2}\right) \\ d \notin E\left(y_{u}\right) \\ y_{u}<y_{2}}} \tilde{s}_{-r, s}(d), \ldots, \sum_{\substack{d \in E\left(y_{n}\right) \\ d \notin E\left(y_{u}\right) \\ y_{u}<y_{n}}} \tilde{s}_{-r, s}(d)\right)
$$

and $E=\left(e_{i j}\right)_{m \times n}$ so that;

$$
e_{i j}= \begin{cases}1, & \text { if } y_{j} \in E\left(t_{i}\right) \\ 0, & \text { otherwise }\end{cases}
$$


Then,

$$
\begin{aligned}
& \left(E A_{-r} E^{T}\right)_{i j} \approx \sum_{k=1}^{n}\left(e_{i k}\left(\sum_{\substack{d \in E\left(y_{k}\right) \\
d \notin E\left(y_{u}\right) \\
y_{u}<y_{k}}} \tilde{s}_{-r, s}(d)\right) e_{j k}\right) \approx \sum_{\substack{y_{k} \in E\left(t_{i}\right) \\
y_{k} \in E\left(t_{j}\right)}}\left(\sum_{\substack{d \in E\left(y_{k}\right) \\
d \notin E\left(y_{u}\right) \\
y_{u}<y_{k}}} \tilde{s}_{-r, s}(d)\right) \\
& \approx \sum_{y_{k} \in E\left(\left(t_{i}, t_{j}\right)_{p}\right)}\left(\sum_{\substack{d \in E\left(y_{k}\right) \\
d \in E\left(y_{u}\right) \\
y_{u}<y_{k}}} \widetilde{s}_{-r, s}(d)\right) \approx\left(t_{i}, t_{j}\right)_{p}{ }^{-r} \text {. }
\end{aligned}
$$

Note: The reciprocal power $G C D P$-matrices could be easily written as $A_{-r} B_{-r}$ and $A_{-r} A_{-r}^{T}$ in a similar manner done above.

Example 4. If $T \approx\left\{1,1+x, 1+x^{3}\right\}$ is a P-ordered gcd-closed set over $\mathbb{Z}_{2}[x]$, then the reciprocal of $\left(T^{2}\right)_{p}$ is

$$
1 /\left(T^{2}\right)_{p} \approx\left[\begin{array}{ccc}
1 & 1 & 1 \\
1 & \frac{1}{1+x^{2}} & \frac{1}{1+x^{2}} \\
1 & \frac{1}{1+x^{2}} & \frac{1}{1+x^{6}}
\end{array}\right]
$$

and is factored as follows:

$$
E A_{-2} E^{T} \approx\left[\begin{array}{ccc}
1 & 0 & 0 \\
1 & 1 & 0 \\
1 & 1 & 1
\end{array}\right]\left[\begin{array}{ccc}
1 & 0 & 0 \\
0 & \frac{-x^{2}}{1+x^{2}} & 0 \\
0 & 0 & \frac{x^{4}-x^{2}}{1+x^{6}}
\end{array}\right]\left[\begin{array}{ccc}
1 & 1 & 1 \\
0 & 1 & 1 \\
0 & 0 & 1
\end{array}\right] \approx\left[\begin{array}{ccc}
1 & 1 & 1 \\
1 & \frac{1}{1+x^{2}} & \frac{1}{1+x^{2}} \\
1 & \frac{1}{1+x^{2}} & \frac{1}{1+x^{6}}
\end{array}\right]
$$

or,

$$
A_{-2} B_{-2} \approx\left[\begin{array}{ccc}
1 & 0 & 0 \\
1 & \frac{-x^{2}}{1+x^{2}} & 0 \\
1 & \frac{-x^{2}}{1+x^{2}} & \frac{x^{4}-x^{2}}{1+x^{6}}
\end{array}\right]\left[\begin{array}{ccc}
1 & 1 & 1 \\
0 & 1 & 1 \\
0 & 0 & 1
\end{array}\right] \approx\left[\begin{array}{ccc}
1 & 1 & 1 \\
1 & \frac{1}{1+x^{2}} & \frac{1}{1+x^{2}} \\
1 & \frac{1}{1+x^{2}} & \frac{1}{1+x^{6}}
\end{array}\right]
$$

or,

$$
A_{-2} A_{-2}^{T} \approx\left[\begin{array}{ccc}
1 & 0 & 0 \\
1 & \sqrt{\frac{-x^{2}}{1+x^{2}}} & 0 \\
1 & \sqrt{\frac{-x^{2}}{1+x^{2}}} & \sqrt{\frac{x^{4}-x^{2}}{1+x^{6}}}
\end{array}\right]\left[\begin{array}{ccc}
1 & 1 & 1 \\
0 & \sqrt{\frac{-x^{2}}{1+x^{2}}} & \sqrt{\frac{-x^{2}}{1+x^{2}}} \\
0 & 0 & \sqrt{\frac{x^{4}-x^{2}}{1+x^{6}}}
\end{array}\right] \approx\left[\begin{array}{ccc}
1 & 1 & 1 \\
1 & \frac{1}{1+x^{2}} & \frac{1}{1+x^{2}} \\
1 & \frac{1}{1+x^{2}} & \frac{1}{1+x^{6}}
\end{array}\right] .
$$

\subsection{Determinant of reciprocal power $G C D P$-matrices on $G C D$-closed sets over a UFD}

Theorem 8. Let $R$ be a UFD and $P \approx\left\{p_{1}, p_{2}, \ldots, p_{i}, \ldots\right\}$ be a complete prime residue system of $R$, and $T \approx\left\{t_{1}, t_{2}, \ldots, t_{m}\right\}$ is a gcd-closed $P$-ordered set of non-zero non-associate elements 
in R. Then,

$$
\operatorname{det}\left(1 /\left(T^{r}\right)_{p}\right) \approx \prod_{i=1}^{m}\left(\sum_{\substack{d \in E\left(t_{i}\right), d \notin E\left(t_{u}\right) \\ t_{u}<t_{i}}} \tilde{s}_{-r, s}(d)\right)
$$

Proof. Let $T$ be a $P$-ordered set in $S$. Since $T$ is a gcd-closed set, then $T \approx D=\left\{y_{1}, y_{2}, \ldots, y_{m}\right\}$ and $1 /\left(T^{r}\right)_{p} \approx E A_{-r} E^{T}$ and $E$ is a lower triangular matrix with $\operatorname{det}[E]=1$. Thus,

$$
\operatorname{det}\left(1 /\left(T^{r}\right)_{p}\right) \approx \operatorname{det}\left(E A_{-r} E^{T}\right) \approx \operatorname{det}\left(A_{-r}\right) \approx \prod_{i=1}^{m}\left(\sum_{\substack{d \in E\left(t_{i}\right) \\ d \notin E\left(t_{u}\right) \\ t_{u}<t_{i}}} \tilde{s}_{-r, s}(d)\right) .
$$

Note that we may prove the above theorem by using the factorizations $1 /\left(T^{r}\right)_{p} \approx A_{-r} B_{-r}$ and $1 /\left(T^{r}\right)_{p} \approx A_{-r} A_{-r}^{T}$.

\section{Inverses of power $G C D P$-matrices on $G C D$-closed sets over a UFD}

Theorem 9. Let $R$ be a UFD and $P \approx\left\{p_{1}, p_{2}, \ldots, p_{i}, \ldots\right\}$ be a complete prime residue system of $R$, and $T \approx\left\{t_{1}, t_{2}, \ldots, t_{m}\right\}$ is a gcd-closed P-ordered set of non-zero non-associate elements in $R$. Then, the inverse of $\left(T^{r}\right)_{p}$ is $\left(T^{r}\right)_{p}^{-1}=\left(t_{i j}\right)_{m \times m}$ such that

$$
t_{i j}=\sum_{t_{i} \in E\left(t_{k}\right), t_{j} \in E\left(t_{k}\right)}\left(\frac{\mu_{p}\left(\frac{t_{k}}{t_{i}}\right) \mu_{p}\left(\frac{t_{k}}{t_{j}}\right)}{\sum_{\substack{d \in E\left(t_{k}\right), d \notin E\left(t_{u}\right) \\ t_{u}<t_{k}}} \tilde{s}_{r, s}(d)}\right) .
$$

Proof. Let $F \approx E^{-1}$, such that

$$
f_{i j}=\left\{\begin{array}{cl}
\mu\left(\frac{t_{i}}{t_{j}}\right), & \text { if } t_{j} \in E\left(t_{i}\right) \\
0, & \text { otherwise }
\end{array}\right.
$$

then

$$
\begin{aligned}
t_{i j} & \approx\left(E A_{r} E^{T}\right)_{i j}^{-1} \approx\left(\left(E^{T}\right)^{-1}\left(A_{r}\right)^{-1}(E)^{-1}\right)_{i j} \approx\left(F^{T}\left(A_{r}\right)^{-1} F\right)_{i j} \approx \sum_{k=1}^{m} f_{k i} \frac{1}{a_{k k}} f_{k j} \\
& \left.\approx \sum_{\substack{t_{i} \in E\left(t_{k}\right) \\
t_{j} \in E\left(t_{k}\right)}}\left(\mu_{p}\left(\frac{t_{k}}{t_{i}}\right) \frac{1}{\sum_{\substack{d \in E\left(t_{k}\right) \\
d \notin E\left(t_{u}\right)}} \tilde{s}_{r, s}(d)} \mu_{p}\left(\frac{t_{k}}{t_{j}}\right)\right) \approx \sum_{\substack{t_{i} \in E\left(t_{k}\right) \\
t_{j} \in E\left(t_{k}\right)}} \frac{\mu_{p}\left(\frac{t_{k}}{t_{i}}\right) \mu_{p}\left(\frac{t_{k}}{t_{j}}\right)}{\sum_{\substack{d \in E\left(t_{k}\right) \\
\tilde{s}_{r, s}(d)}}\left(t_{u}\right)}\right)
\end{aligned}
$$


Example 5. If $T \approx\{1,1+i, 2\}$ is a P-ordered gcd-closed set of the $\mathbb{Z}[i]$, then, by computing the entries of $\left(T^{2}\right)_{p}^{-1}$ we get:

$$
\begin{aligned}
& t_{11}=\sum_{1 \in E\left(t_{k}\right)}\left(\frac{\mu_{p}\left(\frac{t_{k}}{1}\right) \mu_{p}\left(\frac{t_{k}}{1}\right)}{\sum_{\substack{d \in E\left(t_{k}\right) \\
d \notin E\left(t_{u}\right) \\
t_{u}<t_{k}}} \widetilde{s}_{2, s}(d)}\right)=\frac{4}{5}-\frac{2}{5} i=\left(T^{2}\right)_{11}^{-1},
\end{aligned}
$$

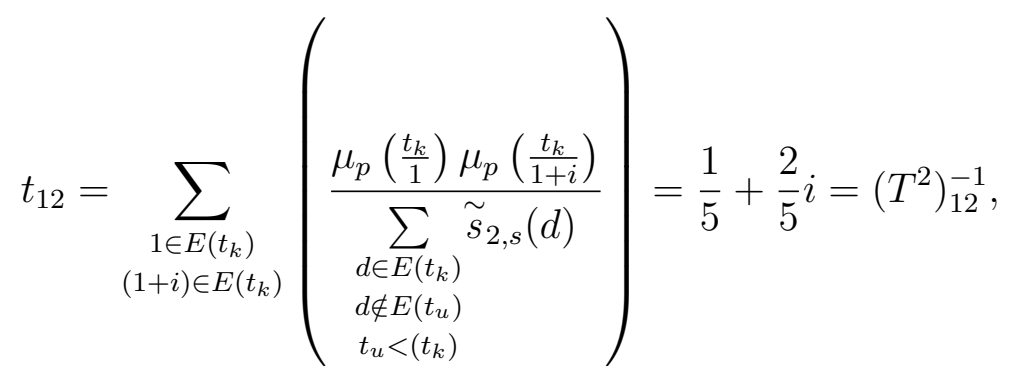

$$
\begin{aligned}
& t_{13}=\sum_{\substack{1 \in E\left(t_{k}\right) \\
(2) \in E\left(t_{k}\right)}}\left(\frac{\mu_{p}\left(\frac{t_{k}}{1}\right) \mu_{p}\left(\frac{t_{k}}{2}\right)}{\sum_{\substack{d \in E\left(t_{k}\right) \\
d \notin E\left(t_{u}\right) \\
t_{u}<\left(t_{k}\right)}}^{\widetilde{s}_{2, s}(d)}}\right)=0=\left(T^{2}\right)_{13}^{-1}, \\
& t_{21}=\sum_{\substack{2 \in E\left(t_{k}\right) \\
1 \in E\left(t_{k}\right)}}\left(\frac{\mu_{p}\left(\frac{t_{k}}{2}\right) \mu_{p}\left(\frac{t_{k}}{1}\right)}{\sum_{\substack{d \in E\left(t_{k}\right) \\
d \notin E\left(t_{u}\right) \\
t_{u}<\left(t_{k}\right)}}^{\widetilde{s}_{2, s}(d)}}\right)=\frac{1}{5}+\frac{2}{5} i=\left(T^{2}\right)_{21}^{-1}, \\
& t_{22}=\sum_{(1+i) \in E\left(t_{k}\right)}\left(\frac{\mu_{p}\left(\frac{t_{k}}{(1+i)}\right) \mu_{p}\left(\frac{t_{k}}{(1+i)}\right)}{\sum_{\substack{d \in E\left(t_{k}\right) \\
d \notin E\left(t_{u}\right) \\
t_{u}<\left(t_{k}\right)}} \tilde{s}_{2, s}(d)}\right)=-\frac{3}{10} i=\left(T^{2}\right)_{22}^{-1} \text {, } \\
& t_{23}=\sum_{\substack{(1+i) \in E\left(t_{k}\right) \\
2 \in E\left(t_{k}\right)}}\left(\frac{\mu_{p}\left(\frac{t_{k}}{(1+i)}\right) \mu_{p}\left(\frac{t_{k}}{2}\right)}{\sum_{\substack{d \in E\left(t_{k}\right) \\
d \notin E\left(t_{u}\right) \\
t_{u}<\left(t_{k}\right)}} \widetilde{s}_{2, s}(d)}\right)=-\frac{1}{5}-\frac{1}{10} i=\left(T^{2}\right)_{23}^{-1} \text {, }
\end{aligned}
$$




$$
\begin{aligned}
& t_{31}=\sum_{\substack{2 \in E\left(t_{k}\right) \\
1 \in E\left(t_{k}\right)}}\left(\frac{\mu_{p}\left(\frac{t_{k}}{1}\right) \mu_{p}\left(\frac{t_{k}}{2}\right)}{\sum_{\substack{d \in E\left(t_{k}\right) \\
d \notin E\left(t_{u}\right) \\
t_{u}<\left(t_{k}\right)}} \widetilde{s}_{2, s}(d)}\right)=0=\left(T^{2}\right)_{31}^{-1}, \\
& t_{32}=\sum_{\substack{2 \in E\left(t_{k}\right) \\
(1+i) \in E\left(t_{k}\right)}}\left(\frac{\mu_{p}\left(\frac{t_{k}}{2}\right) \mu_{p}\left(\frac{t_{k}}{(1+i)}\right)}{\sum_{\substack{d \in E\left(t_{k}\right) \\
d \notin E\left(t_{u}\right) \\
t_{u}<\left(t_{k}\right)}} \tilde{s}_{2, s}(d)}\right)=-\frac{1}{5}-\frac{1}{10} i=\left(T^{2}\right)_{32}^{-1} \text {, }
\end{aligned}
$$

and

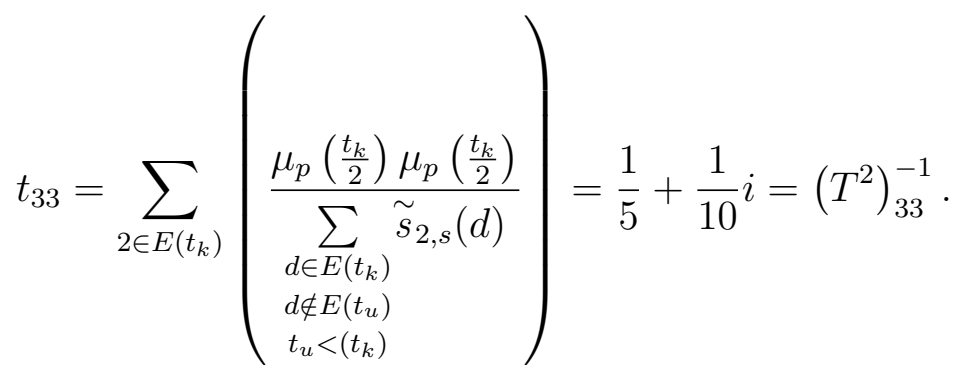

Hence,

$$
\left(T^{2}\right)_{p}\left(T^{2}\right)_{p}^{-1} \approx\left[\begin{array}{ccc}
1 & 1 & 1 \\
1 & 2 i & 2 i \\
1 & 2 i & 4
\end{array}\right]\left[\begin{array}{ccc}
\frac{4}{5}-\frac{2}{5} i & \frac{1}{5}+\frac{2}{5} i & 0 \\
\frac{1}{5}+\frac{2}{5} i & -\frac{3}{10} i & -\frac{1}{5}-\frac{1}{10} i \\
0 & -\frac{1}{5}-\frac{1}{10} i & \frac{1}{5}+\frac{1}{10} i
\end{array}\right]=\left[\begin{array}{ccc}
1 & 0 & 0 \\
0 & 1 & 0 \\
0 & 0 & 1
\end{array}\right]
$$

\section{Power $L C M P$-matrices on $G C D$-closed sets over a UFD}

\subsection{Factorizations of power $L C M P$-matrices}

\section{on $G C D$-closed sets over a UFD}

Theorem 10. Let $R$ be a UFD and $P \approx\left\{p_{1}, p_{2}, \ldots, p_{i}, \ldots\right\}$ be a complete prime residue system of $R$, and $T \approx\left\{t_{1}, t_{2}, \ldots, t_{m}\right\}$ be a gcd-closed $P$-ordered set of non-zero non-associate elements in R. The power LCM matrix can be decomposed, up to associates, as

$$
\left[T^{r}\right]_{p} \approx D_{r} E A_{-r} E^{T} D_{r}
$$

Proof. Let $D=\left\{y_{1}, y_{2}, \ldots, y_{n}\right\}$ be the smallest gcd-closed set containing $T$ in $R$, and $E(x)$ be a complete set of distinct non-associate divisors $d$ of $t$ in $R$. Define the following matrices: $\left(A_{-r}\right)_{n \times n}$ is a diagonal matrix such that:

$$
A_{-r} \approx \operatorname{diag}\left(\sum_{\substack{d \in E\left(y_{1}\right) \\ d \notin E\left(y_{u}\right) \\ y_{u}<y_{1}}} \tilde{s}_{-r, s}\left(y_{1}\right), \sum_{\substack{d \in E\left(y_{2}\right) \\ d \notin E\left(y_{u}\right) \\ y_{u}<y_{2}}} \tilde{s}_{-r, s}\left(y_{2}\right), \ldots, \sum_{\substack{d \in E\left(y_{n}\right) \\ d \notin E\left(y_{u}\right) \\ y_{u}<y_{n}}} \tilde{s}_{-r, s}\left(y_{n}\right)\right)
$$


$E=\left(e_{i j}\right)_{m \times n}$ such that:

$$
e_{i j}= \begin{cases}1, & \text { if } y_{j} \in E\left(t_{i}\right) \\ 0, & \text { otherwise }\end{cases}
$$

and $D_{r} \approx \operatorname{diag}\left(t_{1}^{r}, t_{2}^{r}, \ldots, t_{m}^{r}\right)$. So,

$$
\left(D_{r} E A_{-r} E^{T} D_{r}\right)_{i j} \approx\left(D_{r}\left(T^{-r}\right) D_{r}\right) \approx t_{i}^{r}\left(T^{-r}\right)_{i j} t_{j}^{r} \approx \frac{t_{i}^{r} t_{j}^{r}}{\left(t_{i}, t_{j}\right)^{r}} \approx\left[\left[t_{i}, t_{j}\right]_{p}\right]^{r}
$$

Example 6. If $T \approx\left\{1,1+x, 1+x^{3}\right\}$ is a P-ordered gcd-closed set over $\mathbb{Z}_{2}[x]$, then the LCM power P-matrix $(r=2)$ is:

$$
\left[T^{2}\right]_{p}=\left[\begin{array}{ccc}
1 & 1+x^{2} & 1+x^{6} \\
1+x^{2} & 1+x^{2} & 1+x^{6} \\
1+x^{6} & 1+x^{6} & 1+x^{6}
\end{array}\right]
$$

and the reciprocal GCD power P-matrix is:

$$
1 /\left(T^{2}\right)_{p}=\left[\begin{array}{ccc}
1 & 1 & 1 \\
1 & \frac{1}{1+x^{2}} & \frac{1}{1+x^{2}} \\
1 & \frac{1}{1+x^{2}} & \frac{1}{1+x^{6}}
\end{array}\right]
$$

and

$$
D_{2}=\left[\begin{array}{ccc}
1 & 0 & 0 \\
0 & 1+x^{2} & 0 \\
0 & 0 & 1+x^{6}
\end{array}\right]
$$

Then,

$$
\begin{aligned}
D_{2}\left(1 /\left(T^{2}\right)_{p}\right) D_{2} & \approx\left[\begin{array}{ccc}
1 & 0 & 0 \\
0 & 1+x^{2} & 0 \\
0 & 0 & 1+x^{6}
\end{array}\right]\left[\begin{array}{ccc}
1 & 1 & 1 \\
1 & \frac{1}{1+x^{2}} & \frac{1}{1+x^{2}} \\
1 & \frac{1}{1+x^{2}} & \frac{1}{1+x^{6}}
\end{array}\right]\left[\begin{array}{ccc}
1 & 0 & 0 \\
0 & 1+x^{2} & 0 \\
0 & 0 & 1+x^{6}
\end{array}\right] \\
& \approx\left[\begin{array}{ccc}
1 & 1+x^{2} & 1+x^{6} \\
1+x^{2} & 1+x^{2} & 1+x^{6} \\
1+x^{6} & 1+x^{6} & 1+x^{6}
\end{array}\right] \approx\left[T^{2}\right]_{p} .
\end{aligned}
$$

\subsection{Determinants of power $L C M P$-matrices}

\section{on $G C D$-closed sets over a UFD}

Theorem 11. Let $R$ be a UFD and $P \approx\left\{p_{1}, p_{2}, \ldots, p_{i}, \ldots\right\}$ be a complete prime residue system of $R$, and $T \approx\left\{t_{1}, t_{2}, \ldots, t_{m}\right\}$ be a gcd-closed P-ordered set of non-zero non-associate elements in R. Then,

$$
\operatorname{det}\left[T^{r}\right]_{p} \approx \prod_{i=1}^{m}\left(\left(\sum_{\substack{d \in E\left(y_{i}\right) \\ d \notin E\left(y_{u}\right) \\ y_{u}<y_{i}}} \tilde{s}_{-r, s}\left(t_{i}\right)\right) t_{i}^{2 r}\right) .
$$


Proof. Since $T$ is a gcd-closed set, then $T \approx D=\left\{y_{1}, y_{2}, \ldots, y_{m}\right\}$ and $\left[T^{r}\right] \approx D_{r} E A_{-r} E^{T} D_{r}$, where $E$ is a lower triangular matrix with diagonal entry $e_{i i}=1$, i.e., $\operatorname{det}[E]=1$. Thus,

$$
\begin{aligned}
\operatorname{det}\left[T^{r}\right]_{p} & \approx \operatorname{det}\left(D_{r} E A_{-r} E^{T} D_{r}\right) \\
& \approx \operatorname{det}\left(D_{r}\right) \operatorname{det}(E) \operatorname{det}\left(A_{-r}\right) \operatorname{det}\left(E^{T}\right) \operatorname{det}\left(D_{r}\right) \\
& \approx \prod_{i=1}^{m} t_{i}^{r} \times \operatorname{det}\left(A_{-r}\right) \times \prod_{i=1}^{m} t_{i}^{r} \approx \prod_{i=1}^{m}\left(\left(\sum_{\substack{d \in E\left(y_{i}\right) \\
d \notin E\left(y_{u}\right) \\
y_{u}<y_{i}}} \tilde{s}_{-r, s}\left(t_{i}\right)\right) t_{i}^{2 r}\right) .
\end{aligned}
$$

Example 7. If $T=\left\{1,1+x, 1+x^{3}\right\}$ is a P-ordered gcd-closed set over $\mathbb{Z}_{2}[x]$, then the determinant of the power LCM P-matrix with $r=2$ is

$$
\begin{aligned}
\operatorname{det}\left[T^{2}\right]_{p} & \approx \prod_{i=1}^{3}\left(\sum_{\left.\begin{array}{l}
d \in E\left(y_{i}\right) \\
d \notin E\left(y_{u}\right) \\
y_{u}<y_{i}
\end{array}\right)} \tilde{s}_{-2, s}\left(t_{i}\right) t_{i}^{2 \times 2}\right) \\
& \approx-x^{14}-2 x^{13}-3 x^{10}+3 x^{8}+2 x^{5}+x^{4} \\
& \approx x^{14}-x^{10}+x^{18}-x^{4} .
\end{aligned}
$$

\section{Acknowledgements}

The authors would like to thank the referees for their significant recommendations and remarks that brought about enhancing this paper.

\section{References}

[1] Beslin, S., \& El-Kassar, A. N. (1989). GCD matrices and Smith's determinant over U.F.D., Bull. Number Theory Related Topics, 13, 17-22.

[2] Beslin, S., \& Ligh, S. (1989). Greatest common divisor matrices, Linear Algebra and Its Applications, 118, 69-76.

[3] Beslin, S. (1991). Reciprocal GCD matrices and LCM matrices, Fibonacci Quart., 20, 71-274.

[4] Beslin, S., \& Ligh, S. (1989). Another generalization of Smith's determinant, Bull Australian Math. Soc., 40, 413-415. 
[5] Beslin, S., \& Ligh, S. (1992). GCD-closed sets and the determinants of GCD matrices, Fibonacci Quart., 30, 157-160.

[6] Borque, K., \& Ligh, S. (1992). On GCD and LCM matrices, Linear Algebra and Its Applications, 174, 65-74.

[7] Chun, S. Z. (1996). GCD and LCM Power Matrices, Fibonacci Quart., 43, 290-297.

[8] El-Kassar, A. N., Awad, Y. A., \& Habre, S. S. (2009). GCD and LCM matrices on factor closed sets defined in principle ideal domains, Journ. Math. and Stat., 5, 342-347.

[9] El-Kassar, A. N., Habre, S. S., \& Awad, Y. A. (2010). GCD Matrices Defined on gcd-closed Sets in a PID, International Journal of Applied Mathematics, 23, 571-581.

[10] Haukkanen, P. (1997). On Smith's determinant, Linear Algebra and Its Applications, 258, 251-269.

[11] Haukkanen, P., \& Sillanpää, J. (1997). On some analogues of the Bourque-Ligh conjecture on LCM matrices, Notes on Number Theory and Discrete Mathematics, 3 (1), 52-57.

[12] Hong, S. (1998). On LCM matrices on GCD-closed sets (English summary), Southeast Asian Bull. Math., 22, 381-384.

[13] Hong, S. (1998). Bounds for determinant of matrices associated with classes of arithmetical functions, Linear Algebra and Its Applications, 281, 311-322.

[14] Hong, S. (2004). Asymptotic behavior of eigen values of GCD power matrices, Glasgow Math. J., 46, 551-569.

[15] Hong, S., Zhou, X., \& Zhao, J. (2009). Power GCD Matrices for a UFD, Algebra Colloquium, 16, 71-78.

[16] Li, Z. (1990). The determinant of a GCD matrices. Lin. and Multilin. Alg., 134, 137-143.

[17] Ligh, S. (1988). Generalized Smith's determinant, Lin. and Multilin. Alg., 22, 305-306.

[18] Smith, H. J. S. (1875/76). On the value of a certain arithmetical determinant, Proc. London Math. Soc., 7, 208-212. 